\section{Impacto de la cirugía plástica mexicana en revistas internacionales: análisis a 5 años}

\author{
Impact of Mexican plastic surgery in international magazines: 5-year analysis
}

\author{
Dr. José E Telich-Tarriba,* Dra. Andrea Nachón-Acosta
}

Palabras clave: Cirugía plástica, análisis bibliométrico, publicaciones científicas, impacto,

autores mexicanos, revistas internacionales.

Keywords:

Plastic surgery, bibliometric analysis, scientific publications,

impact, Mexican authors, international journals.

* Departamento de Cirugía Plástica y Reconstructiva. Hospital Ángeles Pedregal. Ciudad de México, México.

‡ Médico Residente de Cirugía General.

Departamento de Cirugía General, Unidad Médica de Alta Especialidad «Lic. Adolfo Ruiz Cortines», Instituto Mexicano del Seguro Social. Veracruz,

México.

Recibido:

27 agosto 2020 Aceptado para publicar: 14 septiembre 2020

\section{RESUMEN}

La publicación científica es una de las formas más efectivas e importantes en la que los médicos pueden compartir hallazgos, experiencias clínicas y actualizaciones con la finalidad de hacer medicina basada en evidencia. En cirugía plástica existe literatura que analiza la productividad científica en el ámbito mundial, pero sigue desconocida la productividad científica de cirujanos mexicanos en revistas de alto impacto. Por este motivo, se realizó una búsqueda de los artículos publicados en un periodo de cinco años en las revistas con mayor factor de impacto relacionados con el campo de la cirugía plástica, en los que se buscó que alguno de los autores perteneciera a alguna institución mexicana. Se obtuvieron como resultados 61 artículos, los cuales fueron publicados en 13 de las 20 revistas de mayor impacto, participaron 144 autores. Concluimos que sería recomendable que los programas académicos y asociaciones nacionales promuevan estrategias que incentiven la publicación científica en revistas de alto nivel, con el fin de incrementar nuestro impacto y la adquisición de conocimientos que beneficien la práctica médica.

\section{ABSTRACT}

Academic publishing is one of the most effective and important ways in which physicians can share findings, clinical experiences, and updates for the purpose of performing evidence-based medicine. In plastic surgery, there is literature that analyzes scientific productivity worldwide, but the scientific productivity of Mexican surgeons in high impact journals remains unknown. For this reason, a search was performed for articles published in a period of five years in the journals with the highest impact related to the field of plastic surgery, in which authors belonged to a Mexican institution. The outcome was 61 articles, which were published in 13 of the 20 journals with the highest impact, 144 authors participated. We conclude that it would be advisable for academic programs and national associations to promote strategies that encourage scientific publication in high level journals, in order to increase our impact and the acquisition of knowledge that benefit medical practice.

\section{INTRODUCCIÓN}

L a publicación científica es una de las maneras más efectivas en las que los médicos podemos compartir hallazgos en investigación y experiencias clínicas o quirúrgicas con el fin de hacer medicina basada en evidencia y mejorar el manejo de los pacientes. ${ }^{1}$ La publicación de artículos en revistas internacionales de prestigio permite que los autores o sus instituciones alcancen un mayor número de lectores y sus ideas tengan mayor impacto. ${ }^{2}$
De acuerdo con las estadísticas de la International Society of Aesthetic Plastic Surgery (ISAPS), en México se realizan el $4.5 \%$ del total mundial de cirugías estéticas. En el país se cuenta con aproximadamente 1,600 cirujanos plásticos activos, egresados de 14 programas universitarios certificados. ${ }^{3,4}$

Sería esperado que un país con un volumen tan elevado de cirujanos y procedimientos publicara un número importante de artículos científicos; sin embargo, este no ha sido el caso. Un reporte por Navia y colaboradores en 2019

Citar como: Telich-Tarriba JE, Nachón-Acosta A. Impacto de la cirugía plástica mexicana en revistas internacionales: análisis a 5 años. Cir Plast. 2020; 30 (2): 74-77. https://dx.doi.org/10.35366/97673 
informó que México es apenas el tercer productor de artículos dentro de la Federación Iberolatinoamericana de Cirugía Plástica, ${ }^{5}$ mientras que en un análisis bibliométrico internacional de Rymer, nuestro país no figura dentro de los primeros 20 lugares. ${ }^{6}$

La bibliometría es el proceso de analizar el contenido y las citas de los artículos con el fin de cuantificar tendencias en el tipo de publicación, temas, autores, instituciones de origen y la forma en la que se difunden los datos. También destacan las carencias en la literatura actualmente disponible y sirve de guía para la información futura.

Existen publicaciones que analizan tendencias e impacto en la literatura en cirugía plástica en el ámbito mundial; sin embargo, a la fecha sigue siendo desconocida la productividad científica de cirujanos mexicanos en revistas de alto impacto. El objetivo de este trabajo es cuantificar las contribuciones de México en las principales revistas internacionales relacionadas con el campo de la cirugía plástica.

\section{MATERIAL Y MÉTODO}

Se realizó una búsqueda computarizada en MEDLINE de artículos publicados entre el 1 de enero de 2013 y el 31 de diciembre de 2018 en alguna de las 20 revistas con mayor factor de impacto relacionadas con el campo de la cirugía plástica, en los que se identificara que alguno de los autores pertenecía a alguna institución mexicana.

Las revistas incluidas fueron seleccionadas de acuerdo al factor de impacto reportado por Science Citation Reports en 2018. Las revistas con mayor factor de impacto fueron las siguientes: Plastic and Reconstructive Surgery, Aesthetic Surgery Journal, JAMA facial Plastic Surgery, Burns and Trauma, Hand Surgery (European Volume), Journal of Plastic Reconstructive and Aesthetic Surgery, Burns, Hand Surgery (American Volume), Microsurgery, Journal of Cranio-maxillofacial Surgery, Journal of Reconstructive Microsurgery, Journal of Burn Care and Research, Cleft palate Craniofacial Journal, Annals of Plastic Surgery, Aesthetic Plastic Surgery, Clinics in Plastic Surgery, Journal of Plastic Surgery and Hand Surgery, Seminars in Plastic
Surgery, Journal of Craniofacial Surgery y Plastic surgery (Canada).

Los artículos identificados fueron exportados a una hoja de datos en la que se incluyó nombre del artículo, revista, año de publicación, autores, tipo de artículo (investigación original, reporte de caso, carta al editor, revisión), tema (cirugía estética o reconstructiva) y factor de impacto de la revista.

La información se analizó utilizando medidas de tendencia central para variables continuas y totales y porcentajes para variables categóricas.

\section{RESULTADOS}

En total se incluyeron 61 artículos publicados en 13 de las 20 revistas. Participaron 144 autores de 23 instituciones, distribuidas en seis estados del país. La entidad con mayor número de publicaciones fue la Ciudad de México con 30 (49.2\%), seguida por Jalisco (16 publicaciones: $26.2 \%$ ). La institución con mayor número de publicaciones fue el Hospital General «Dr. Manuel Gea González» con 15 (24.6\%), seguido por el Instituto Jalisciense de Cirugía Reconstructiva (IJCR) con seis publicaciones $(9.8 \%)$.

En promedio, se publicaron 10.1 artículos por año (rango 5-15). Las revistas con más publicaciones fueron el Journal of Craniofacial Surgery y Aesthetic Plastic Surgery, con 12 artículos en cada una (19.6\%), seguidos por Plastic and Reconstructive Surgery con 10 publicaciones (16.4\%).

Los artículos más comunes fueron los editoriales (15: 24.6\%), estudios transversales (10: $16.4 \%)$, reportes de caso (9: $14.7 \%$ ) y series de casos (9: 14.7\%). El nivel de evidencia de la mayor parte de los artículos fue 5 .

El promedio de citas de cada artículo fue de $5.0 \pm 8.7$ (rango 0-57). El artículo más citado fue Report on mortality from gluteal fat grafting: recommendations from the ASERF task force.

La cirugía craneofacial fue el tema más abordado (16: $26.2 \%$ ), seguido por la microcirugía (11: 18\%), cirugía mamaria $(7: 11.4 \%)$ y cirugía de contorno corporal (7: $11.4 \%$ ).

Cada artículo tuvo un promedio de $3.7 \pm$ 2.7 coautores (rango 1-11). Los autores con mayor número de publicaciones fueron Lázaro 
Cárdenas Camarena con seis (9.9\%), Alexander Cárdenas, Eric Santamaría y José Abel de la Peña con cuatro artículos cada uno (6.5\%).

\section{DISCUSIÓN}

El análisis de las publicaciones científicas constituye una herramienta fundamental para poder conocer la divulgación del desarrollo científico y técnicas quirúrgicas novedosas. ${ }^{2,7}$ En los últimos 30 años, se ha observado un incremento del $700 \%$ en la cantidad de publicaciones relacionadas con cirugía plástica, va de la mano de grandes avances en investigación básica, clínica y tecnológica, paralelo al progreso de otras ramas de la ciencia y tecnología ${ }^{8}$ y el papel cada vez más relevante en la medicina basada en evidencia en cirugía plástica. ${ }^{9}$

De acuerdo con los reportes anuales de ISAPS, México es uno de los cinco países con mayor número de procedimientos realizados en el mundo, ${ }^{3}$ contando con un gran número de programas de entrenamiento y de cirujanos activos. Sin embargo, a pesar de esto, se ha observado que la cantidad de publicaciones en cirugía plástica en nuestro país es de 27 al año, ${ }^{1}$ lo que nos coloca entre las especialidades con menor número de manuscritos en revistas.

Los estudios bibliométricos son cada vez más comunes en la literatura internacional y se han realizado para evaluar múltiples especialidades, permitiendo conocer los temas de interés, niveles de evidencia, cantidad de citas o tendencias de publicación en general. ${ }^{5}$ Existen iniciativas similares enfocadas a la evaluación de la cirugía plástica en forma global, la gran mayoría de origen norteamericano o europeo. Hasta donde es de nuestro conocimiento, éste es el primer estudio de su tipo enfocado exclusivamente en cirugía plástica mexicana.

Durante el periodo de estudio, se encontraron 61 publicaciones de autores mexicanos, cifra sumamente baja en comparación con otros países. Un estudio previo por Navia y colaboradores encontró que México es el tercer productor de artículos relacionados con la cirugía plástica dentro de los miembros de la Federación Ibero-latinoamericana de Cirugía Plástica, publicando 60\% menos que España y Brasil. ${ }^{5}$ En un estudio bibliométrico internacional de Rymer y Choa, México no figura dentro de los primeros 20 lugares de producción científica en cirugía plástica, a diferencia de otras naciones en desarrollo como Turquía, India o Grecia. ${ }^{6}$

Esta situación parece ser una constante en múltiples áreas científicas en nuestro país; CONACyT reportó que entre 2011 y 2015, la participación de la producción mexicana respecto al total mundial fue de $0.60 \%$, con el área de medicina clínica como una de las menos productivas..$^{10}$ La baja productividad puede sin duda estar asociada con la limitación de recursos que tiene nuestro país y una baja difusión en estrategias de desarrollo de artículos científicos. ${ }^{1}$

A pesar de estos hallazgos, hay factores alentadores a tomar en cuenta que pueden servir de guía o ejemplo a seguir. Las instituciones con mayor número de publicaciones destacan por ser centros académicos dinámicos, con presencia de médicos residentes e investigadores activos, lo que se traduce en una mayor capacidad de producción científica con respecto a clínicas o cirujanos en prácticas privadas. Otro hallazgo que vale la pena mencionar es que el título con mayor número de citas fue el Report on mortality from gluteal fat grafting: recommendations from the ASERF task force, reflejando que las colaboraciones internacionales actualmente son una oportunidad excelente para la generación de artículos de alto impacto. ${ }^{11}$

El presente trabajo cuenta con algunas limitaciones, como únicamente realizar la búsqueda en revistas de alto impacto e ignorar grandes bases de datos como MEDLINE o Scopus, o ignorar revistas latinoamericanas y artículos en español. Sin embargo, el enfocarse en revistas de alto impacto nos permite evaluar mejor el impacto internacional de nuestra especialidad.

\section{CONCLUSIONES}

Es recomendable que, en un futuro, los programas académicos y asociaciones nacionales de cirugía plástica establezcan y promuevan estrategias que incentiven la publicación de artículos en revistas de alto nivel, a fin de incrementar nuestro impacto y acompañar el liderazgo que 
la cirugía plástica mexicana tiene en múltiples esferas internacionales.

\section{REFERENCIAS}

1. Vilchis-López R, Malagón-Hidalgo H, Padilla-Piña J, Fentanes-Vera A. Strategy to increase the number of scientific publications in medical residency. Cir Plast 2017; 27 (3): 99-106.

2. Cuenca-Pardo J, Álvarez-Díaz CJ. La importancia de publicar un artículo médico. Cir Plast 2013; 23 (3): 133-135.

3. La Sociedad Internacional de Cirugía Plástica y Estética (ISAPS) 2018. Disponible en: https://www.isaps.org/ wp-content/uploads/2018/11/2017-Global-SurveyPress-Release_SP.pdf

4. Directorio de Cirujanos Plásticos Certificados por AMCPER. Disponible en: http://directorio. cirugiaplastica.mx/public/

5. Navia A, López A, González JT, Yáñez G, Searle S. Tendencias y perfil de publicación de los cirujanos plásticos de los 22 países miembros de la FILACP, revisión de los últimos 20 años. Cir Plast Iberolatinoam 2019; 45 (1): 81-90. doi: http://dx.doi.org/10.4321/ s0376-78922019000100013

6. Rymer BC, Choa RM. A worldwide bibliometric analysis of published literature in plastic and reconstructive surgery. J Plast Reconstr Aesthet Surg 2015; 68 (9): 1304-1308. doi: https://doi.org/10.1016/j. bjps.2015.05.024
7. Momeni A, Hunter C, Li AY, Safa B, Wan DC, Kneser U. Opinions on authorship. Ann Plast Surg 2018; 80 (6): 660-663. doi: 10.1097/SAP.0000000000001396

8. Zhang WJ, Ding W, Jiang H, Zhang YF, Zhang JL. National representation in the plastic and reconstructive surgery literature. Ann Plast Surg 2013; 70 (2): 231-234. doi: 10.1097/sap.0b013e3182309982

9. Dolan RT, Zins JE, Morrison CM. The aesthetic surgery literature: do plastic surgeons remain at the cutting edge? Plast Reconstr Surg 2016; 138 (1): 277-287. doi: 10.1097/prs.0000000000002260

10. Informe general del estado de la ciencia, la tecnología y la innovación en México 2015. Disponible en: https://www.siicyt.gob.mx/index.php/transparencia/ informes-conacyt/informe-general-del-estado-dela-ciencia-tecnologia-e-innovacion/informe-general2015/3814-informe-general-2015/file

11. Chi D, Curiel D, Bucknor A et al. Institutional collaboration in plastic surgery research: a solution to resource limitations. Plast Reconstr Surg Global Open 2018; 6 (6): e1822. doi: https://doi.org/10.1097/ GOX.0000000000001822

Correspondencia:

Dr. José E Telich-Tarriba

Hospital Ángeles Pedregal

Camino a Santa Teresa Núm. 1055,

Col. Héroes de Padierna, 10700, alcaldía La Magdalena Contreras, Ciudad de México, México.

E-mail: josetelich@gmail.com 